\title{
Specific Patterns of Spinal Metabolite Ratio Underlying a-Me-5-HT- evoked Pruritus Compared with Compound 48/80 Based on Proton Nuclear Magnetic Resonance Spectroscopy*
}

\author{
Ying-le CHEN ${ }^{1}$, Zhi-gang $\mathrm{HE}^{2}$, Qian WANG ${ }^{3}$, Hong-bing XIANG ${ }^{3}$, Li FAN ${ }^{4 \#}$, Jun XIONG ${ }^{5 \#}$ \\ ${ }^{1}$ Department of Anesthesiology, the First Affiliated Quanzhou Hospital of Fujian Medical University, Quanzhou 362000, China \\ ${ }^{2}$ Emergency Medicine, Tongji Hospital, Tongji Medical College, Huazhong University of Science and Technology, Wuhan \\ 430030, China \\ ${ }^{3}$ Department of Anesthesiology and Pain Medicine, Tongji Hospital, Tongji Medical College, Huazhong University of Science \\ and Technology, Wuhan 430030, China \\ ${ }^{4}$ Department of Orthopedics, Union Hospital, Tongji Medical College, Huazhong University of Science and Technology, Wuhan \\ 430022, China \\ ${ }^{5}$ Hepatobiliary Surgery Center, Union Hospital, Tongji Medical College, Huazhong University of Science and Technology, \\ Wuhan 430022, China
}

(C) The Author(s) 2020

\begin{abstract}
Summary: Mechanisms of pruritus are implicated in the dysregulation of the metabolites in the spinal cord. We investigated pruritus behavioral testing in three groups of young adult male $\mathrm{C} 57 \mathrm{Bl} / 6$ mice, including one group treated with normal saline, while the other groups intradermally injected with $\alpha$-Me-5-HT (histamine-independent pruritogen), compound 48/80 (histaminedependent pruritogen) at the nape skin of the neck, respectively. Proton nuclear magnetic resonance spectroscopy (MRS) was used to compare spinal metabolites from the vertebral cervical among three groups, and to study the association of spinal metabolite ratio and pruritus intensity. The MRS-measured N-acetylaspartate-to-myoinositol ratio (NAA/Ins) was significantly correlated with the number of scratches between normal saline group and 48/80 group or $\alpha$-Me-5-HT group (both $P<0.0001$ ), indicating that NAA/Ins may be a robust surrogate marker of histamine-independent/ dependent pruritogen. There was significant difference in Glu/Ins between normal saline group and 48/80 group $(P=0.017)$, indicating that Glu/Ins may be a surrogate marker of histamine-dependent pruritogen, while GABA/Ins was highly significantly different between normal saline group and $\alpha$-Me-5-HT group $(P=0.008)$, suggesting that GABA/Ins may be a surrogate marker of histamineindependent pruritogen. MRS may reflect the extent of pruritus intensity elicited by $\alpha-\mathrm{Me}-5-\mathrm{HT}$ and compound $48 / 80$ with sensitivity similar to the number of scratches, and above potential markers need to be further validated in pre-clinical and clinical treatment trials.
\end{abstract}

Key words: itch; pruritus intensity; spinal cord; metabolomics; proton nuclear magnetic resonance

Emerging research indicates that individuals with unpleasant pruritus sensation, serving as a physiological self-protective mechanism, have an increased risk of life quality impairment resulting in a significant clinical problem ${ }^{[1-3]}$. The spinal cord is an important neural structure involved in pruritus ${ }^{[4]}$, and alterations of gene expression and metabolic profiles in spinal cord are observed in different itch models ${ }^{[5-8]}$.

Ying-le CHEN, E-mail: cylfj@126.com

\#Corresponding authors, Li FAN, E-mail: fanleeeee@163. com; Jun XIONG, E-mail: oldxiong@qq.com

${ }^{*}$ This work was supported by grants from the National Natural Science Foundation of China (No. 81670240 and No. 81873467) and the Medical Innovation Project in Fujian Province (No. 2017-CX-48).
To our knowledge, magnetic resonance spectroscopy (MRS) has not yet been used to assess the associations of pruritus intensity with positive correlates of spinal metabolite, and it remains relatively unknown whether spinal metabolite ratio predicts pruritus intensity.

Recently, our laboratory has used the proton nuclear MRS to investigate specific patterns of spinal metabolites underlying histamine-independent pruritogen $\alpha$-Me-5-HT-evoked pruritus compared with histamine and capsaicin ${ }^{[7]}$. Results from these studies provide a general strategy for the previously proposed method ${ }^{[9-12]}$. Proton nuclear MRS is a commonly used tool to measure levels of N-acetylaspartate (NAA), glutamate (Glu), creatine (Cr), myo-inositol (Ins), etc. An attractive feature of this neuroimaging technology is 
its ability to offer valuable in-vitro neural information, which allows for the identification of chemical metabolite markers of central nervous system (CNS) activity in a given target tissue ${ }^{[12-14]}$. Furthermore, the use of MRS in different times can provide useful information about the hierarchical distinction of same target tissue. Previous applications of this technique have suggested the glutamate-glutamine/myo-inositol ratio $(\mathrm{Glx} / \mathrm{Ins})$ in the anterior cingulate cortex predicts high neuropathic pain impact after spinal cord injury ${ }^{[15]}$. Taken together, it is possible that a more refined analysis of the metabolic profiles of various target tissues helps reveal the nature of the interactions that give rise to specific behavior states.

Research suggests that the experience of pruritus is anatomically and functionally integrated in the spinal cord which can modulate sensory aspect of itching ${ }^{[16,17]}$. We therefore hypothesized that there existed the association of pruritus intensity and spinal metabolite ratio obtained by MRS. This study aimed to describe the pruritus intensity measured by the number of scratches and the changes of metabolic concentrations in the spinal cord extracts after the injection of different pruritogen compounds, and to compare these spinal metabolite ratios with respect to pruritus intensity.

\section{MATERIALS AND METHODS}

\subsection{Animals}

Young adult male C57B1/6 mice weighing 20-25 $\mathrm{g}$ were purchased from Experimental Animal Center of Tongji Medical College, Huazhong University of Science and Technology. The mice were housed in plastic cages with no more than three animals per cage under a controlled 12/12-h light-dark cycle (light from 7:00 AM to 7:00 PM), with the room temperature kept at $23 \pm 1{ }^{\circ} \mathrm{C}$ and humidity at $55 \% \pm 5 \%$. The mice were given free access to water and food pellets. The study was approved by the Animal Care and Use Committee of Tongji Hospital, Tongji Medical College (No. TJA20150803).

\subsection{Itch Model and Behavior Test}

The 29 mice were randomly divided into three groups: normal saline (NS) group ( $n=9), \alpha$-methyl-5hydroxytryptamine ( $\alpha$-Me-5-HT, histamine-independent pruritogen) group $(n=10)$ and $48 / 80$ (compound 48/80, histamine-dependent pruritogen) group $(n=10)$. Under isoflurane ( $2 \%$ in pure oxygen) anesthesia, the nape of the neck was shaved 3 days before the behavior test. On the 4th day, each mouse was habituated for $15 \mathrm{~min}$ and briefly removed from the container and quickly received subcutaneous injection of $50 \mu \mathrm{L}, 0.6 \mathrm{~g} / \mathrm{L}$ $\alpha$-Me-5-HT, $100 \mu \mathrm{g} / 100 \mu \mathrm{L}$ compound $48 / 80$ or $50 \mu \mathrm{L}$ NS at the nape skin of the neck, and the mice were immediately sent back to the container. As previously reported $^{[7,8,18-20]}$, video cameras were used to record the activities of the mice during the following 30 min postinjection. The times of ticky were recorded, and the difference between the NS group and the experimental groups was obtained.

\subsection{Sample Preparation of Spinal Cord for MRS Study}

After the euthanasia with microwave method which was described in our previous work ${ }^{[7]}$, the mouse spinal cords (C5-C8) were quickly removed from the vertebral cervicals to a $2 \mathrm{~mL}$ EP tube. These tissues were immediately weighed and stored at $-80^{\circ} \mathrm{C}$ for further processing.

Protocols for spinal cord tissue extraction were the same as our previous study ${ }^{[7]}$. The extraction steps were repeated twice with $1200 \mu \mathrm{L} 60 \%$ ethanol to extract the substance. All the supernatants were collected together and desiccated with the centrifugal drying apparatus (Thermo Scientific 2010, Germany). The dried product was successively dissolved with $60 \mu \mathrm{L}$ phosphate buffer containing Trisodium phosphate (TSP) (120 mg/L, 269913-1G, Sigma-Aldrich, USA) and $540 \mu \mathrm{L}$ distilled water, and TSP was set as the internal standard. The solution was centrifuged at $14000 \mathrm{~g}$ for $15 \mathrm{~min}$, and the supernatant was withdrawn and transferred to NMR tube for MRS study.

\subsection{MRS}

All MRS spectra were acquired at $298 \mathrm{~K}$ on a BrukerAvanceIII $600 \mathrm{MHzNMR}$ spectrometerequipped with an inverse cryogenic probe (BrukerBiospin, Germany). In order to assign the metabolites in the ${ }^{1} \mathrm{H}$ NMR spectra, a series of two-dimensional (2D) NMR spectra were collected according to our previous study ${ }^{[7]}$. All the MRS studies were completed using the commercial software TOPSPIN (V3.0, BrukerBiospin, Germany).

\subsection{Statistical Analysis}

Multivariate data analysis was performed with the software SPSS (Version 22) and home-made code NMRSpec in MATLAB. Behavioral data are expressed as the mean \pm standard error of the mean (SEM). Analysis was performed using GraphPad Prism 6.0. Comparisons among the three groups were performed using the one-way analysis of variance (ANOVA), followed by post hoc Dunnett's test. The $P$ values of less than 0.05 were considered statistically significant.

\section{RESULTS}

\subsection{Grouping and Evaluation}

We examined the scratching behaviors in male C57BL/6J mice after subcutaneous injection of pruritic agent $\alpha$-Me-5-HT, compound $48 / 80$ and NS into the nape of the neck. As compared with $\alpha-\mathrm{Me}-5$-HT group, mice in compound $48 / 80$ group exhibited a dramatic increase in scratching behaviors (fig. 1; Mann-Whitney 
test; $P<0.01)$. The pruritus intensity between NS group and compound 48/80 group was significantly different $(P<0.001)$. The pruritus intensity between NS group and $\alpha$-Me-5-HT group was also significantly different $(P<0.001)$.

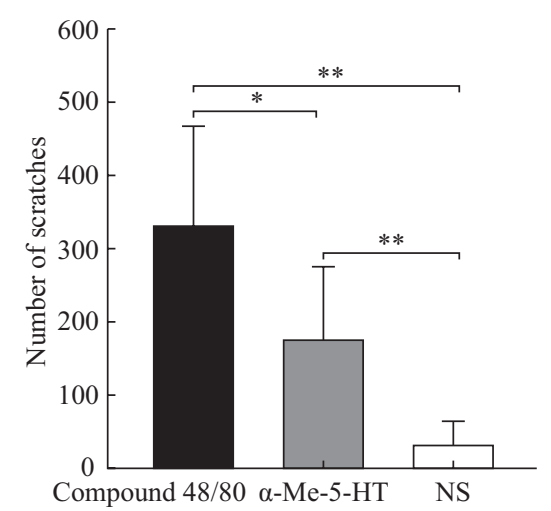

Fig. 1 The total number of scratches during a period of $30 \mathrm{~min}$ in response to subcutaneous injections into the nap of the neck of different compounds in a volume of $50 \mu \mathrm{L}$ Data represents means \pm SEM. ${ }^{*} P<0.01,{ }^{* *} P<0.001$

\subsection{Metabolic Concentration Ratios in Spinal Cord Extracts}

Normalized average MRS spectra of selected metabolites in the spinal cord are illustrated in fig. 2 . The whole correlation matrix of these spinal metabolite concentration ratios is shown in table 1. In the current study, the MRS-measured NAA/Ins was significantly correlated with the pruritus intensity measured by the number of scratches. The pruritus intensity between the NS group and the compound 48/80 group was significantly different $(P<0.001)$. The pruritus intensity between the NS group and $\alpha$-Me-5-HT group was also significantly different $(P<0.001)$. In addition, Glu/Ins was significantly different between the NS group and the compound $48 / 80$ group $(P=0.009$ ), while GABA/ Ins was significantly different between the NS group and the $\alpha$-Me-5-HT group $(P=0.004)$.

\section{DISCUSSION}

The present study investigated the changes of metabolic concentration in spinal cord during NS-, $\alpha$-Me-5-HT-, and compound 48/80-induced pruritic behavior as assessed by MRS, and the association of pruritus intensity and spinal metabolite concentration ratios was compared. The principal findings were collected in the following: (1) as compared with the $\alpha$-Me-5-HT group, the mice in compound 48/80 group exhibited a dramatic increase in scratching behaviors; (2) The MRS-measured NAA/Ins was significantly correlated with the pruritus intensity measured by the number of scratches. The pruritus intensity between the NS group and the compound 48/80 group or $\alpha-\mathrm{Me}-5$ HT group was significantly different (both $P<0.001$ ), indicating NAA/Ins as robust surrogate marker of histamine-independent/dependent pruritogen. (3) Glu/ Ins was significantly different between the NS group and the compound $48 / 80$ group $(P=0.009)$, indicating $\mathrm{Glu} / \mathrm{Ins}$ as a surrogate marker of histamine-dependent pruritogen, whereas GABA/Ins was significantly different between the NS group and the $\alpha-\mathrm{Me}-5-\mathrm{HT}$ group $(P=0.004)$, indicating GABA/Ins as a surrogate marker of histamine-independent pruritogen.

The rapid advancements of MRS and computational frameworks enable the examination of biological processes in unprecedented detail and offer an excellent opportunity to quantify metabolite concentrations of specific brain regions and spinal cord for detecting neuronal activation induced by noxious stimuli. Zeng et al systematically analyzed the host brain proteomes after acute infection with the pseudorabies virus (PRV) and identified a series of potentially PRV-strain-specific regulated proteins with diverse biological functions, suggesting that this MRS study may provide new clues for understanding the neural response of infection and spreading of the virus $^{[21]}$. A study by Wang et al provided evidence of

Table 1 Selected metabolite concentration ratios in the spinal cord among three groups

\begin{tabular}{lcccc}
\hline Metabolite ratio & NS $(n=9)$ & Compound $48 / 80(n=10)$ & $\alpha$-Me-5-HT $(n=10)$ & $P$ \\
\hline Glu/Ins & $1.240 \pm 0.045$ & $1.268 \pm 0.097$ & $1.157 \pm 0.072$ & ${ }^{*}(0.009),{ }^{\Delta}(0.009)$ \\
NAA/Ins & $0.825 \pm 0.011$ & $0.867 \pm 0.056$ & $0.828 \pm 0.059$ & ${ }^{*}(0.043)$ \\
GABA/Ins & $1.396 \pm 0.159$ & $1.322 \pm 0.154$ & $1.196 \pm 0.100$ & ${ }^{\#}(0.004),{ }^{\Delta}(0.044)$ \\
Ala/Ins & $0.517 \pm 0.038$ & $0.490 \pm 0.041$ & $0.463 \pm 0.034$ & ${ }^{*}(0.004)$ \\
Lac/Ins & $4.124 \pm 0.216$ & $3.909 \pm 0.182$ & $3.865 \pm 0.291$ & ${ }^{*}(0.031),{ }^{\#}(0.043)$ \\
Asp/Cr & $0.500 \pm 0.022$ & $0.494 \pm 0.032$ & $0.468 \pm 0.041$ & ${ }^{\#}(0.050)$ \\
Gln/Cr & $0.528 \pm 0.017$ & $0.538 \pm 0.027$ & $0.506 \pm 0.033$ & ${ }^{\Delta}(0.027)$ \\
Glu/Cr & $0.960 \pm 0.051$ & $0.994 \pm 0.073$ & $0.880 \pm 0.112$ & ${ }^{\Delta}(0.015)$ \\
Glx/Cr & $1.550 \pm 0.054$ & $1.589 \pm 0.093$ & $1.457 \pm 0.113$ & ${ }^{\#}(0.037),{ }^{\Delta}(0.010)$ \\
GABA/Cr & $1.074 \pm 0.053$ & $1.034 \pm 0.091$ & $0.909 \pm 0.121$ & ${ }^{\#}(0.001),{ }^{\Delta}(0.018)$ \\
$\mathrm{Ala} / \mathrm{Cr}$ & $0.400 \pm 0.017$ & $0.384 \pm 0.034$ & $0.351 \pm 0.033$ & ${ }^{*}(0.001),{ }^{\Delta}(0.039)$ \\
$\mathrm{Lac} / \mathrm{Cr}$ & $3.194 \pm 0.251$ & $3.069 \pm 0.224$ & $2.924 \pm 0.241$ & ${ }^{\#}(0.028)$ \\
\hline
\end{tabular}

Ins, myo-inositol; Cho, choline; Asp, aspartate; Gln, glutamate; Glu, glutamine; Glx, glutamate and glutamine; NAA, N-acetylaspartate; GABA, gamma amino acid butyric acid; Ala, alanine; Lac, lactate; $\mathrm{Cr}$, creatine

${ }^{*} P<0.05$, compound $48 / 80$ group vs. NS group; ${ }^{\#} P<0.05, \alpha$-Me-5-HT group $v s$. NS group; ${ }^{\wedge} P<0.05, \alpha-M e-5$-HT group $v s$. compound $48 / 80$ group 

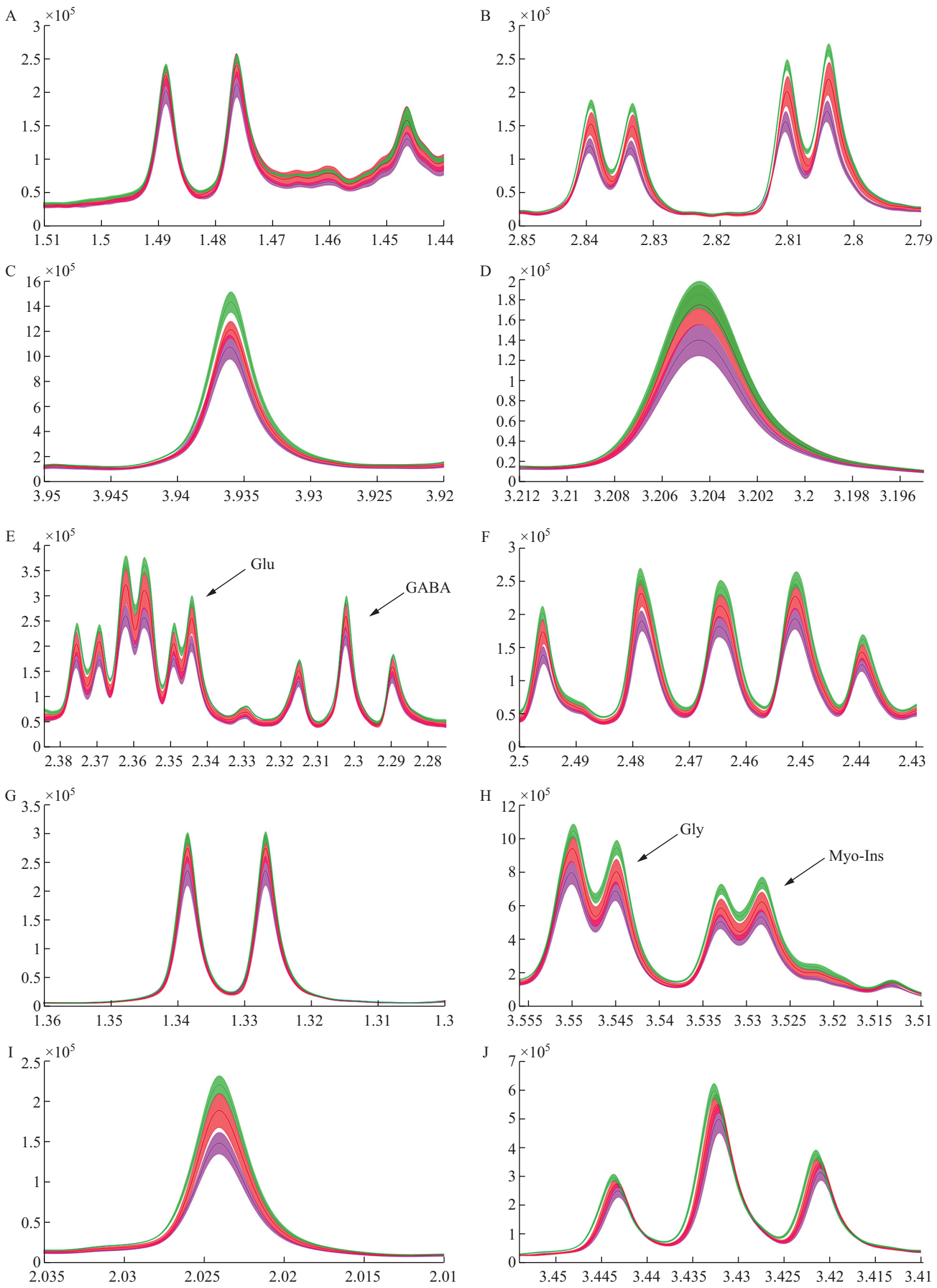

Fig. 2 The difference of normalized spectra of metabolites in lower cervical spinal cord with different itch models in mice A: alanine; B: aspartate; C: choline; D: creatinine; E: GABA and glutamate; F: glutamine; G: lactate; H: myo-inositol and glycine; I: N-acetylaspartate; J: taurine. The spectral line and the width of its shadow represent mean and standard deviation, respectively. NS group: pink line with shadow around; compound 48/80 group: red line with shadow around; $\alpha-\mathrm{Me}-5-\mathrm{HT}$ group: green line with shadow around 
persistent biochemical change of the hypothalamus in patients with episodic cluster headache by MRS, and found low levels of NAA/Cr and $\mathrm{Cho} / \mathrm{Cr}$, suggesting that cluster headache may be related to both neuronal dysfunction and changes in the membrane lipids in the hypothalamus ${ }^{[22]}$.

Pain and itch are two distinct sensations, and share many similarities ${ }^{[16,23]}$. Metabolomics can reveal a correlation between spinal N,N-dimethylsphingosine and neuropathic pain, providing new therapeutic target as a biomarker ${ }^{[24,25]}$. In addition, previous report showed that metabolite concentrations in the anterior cingulate cortex by MRS might be a useful biomarker for severe neuropathic pain to predict the pain intensity ${ }^{[15]}$. Tremendous progress has been made in recent years unravelling spinal mechanisms in acute and chronic pruritus models ${ }^{[26-29]}$. We tried to seek the spinal biomarker to predict the intractable pruritus intensity, and hypothesized that MRS metabolomics could reveal that severe pruritus would be associated with metabolite ratios in the spinal cord, indicating neuronal and/or glial dysfunction. We found that the MRS-measured NAA/Ins was significantly correlated with the pruritus intensity measured by the number of scratches, suggesting that the combination of neuronal dysfunction and proliferation of glia (or glial activation) are underlying mechanisms contributing to the maintenance of severe pruritus. In addition, Glu/Ins was significantly different between the NS group and the compound $48 / 80$ group $(P=0.009)$, suggesting the increased glutamatergic metabolism in combination with glial proliferation or activation, indicating Glu/ Ins as a surrogate marker of histamine-dependent pruritogen. GABA/Ins was significantly different between the NS group and the $\alpha$-Me-5-HT group ( $P=0.004$ ), suggesting the decreased GABAnergic function in combination with glial proliferation or activation, indicating GABA/Ins as a surrogate marker of histamine-independent pruritogen.

In conclusion, this study demonstrated that intense pruritus in mouse affected the tissue levels of amino acids and their ratios in the investigated spinal cord segments, suggesting that alterations in the metabolisms of amino acids might be related to both neuronal dysfunction and glial activation in spinal cord. Three metabolite ratios were found to be associated with the pruritus intensity when stimulated with $\alpha-\mathrm{Me}-5-\mathrm{HT}$ and compound 48/80. Larger animal and human studies will be needed to validate these potential markers of pruritus intensity.

\section{Open Access}

This article is licensed under a Creative Commons Attribution 4.0 International License https://creativecommons.org/licenses/by/4.0/), which permits use, sharing, adaptation, distribution and reproduction in any medium or format, as long as you give appropriate credit to the original author(s) and the source, provide a link to the Creative Commons licence, and indicate if changes were made. The images or other third party material in this article are included in the article's Creative Commons licence, unless indicated otherwise in a credit line to the material. If material is not included in the article's Creative Commons licence and your intended use is not permitted by statutory regulation or exceeds the permitted use, you will need to obtain permission directly from the copyright holder. To view a copy of this licence, visit http://creativecommons.org/licenses/by/4.0/.

\section{Conflict of Interest Statement}

The authors declare no conflicts of interest.

\section{REFERENCES}

1 Meixiong J, Anderson M, Limjunyawong $\mathrm{N}$, et al. Activation of Mast-Cell-Expressed Mas-Related G-Protein-Coupled Receptors Drives Non-histaminergic Itch. Immunity, 2019,50(5):1163-1171

2 Meixiong J, Vasavda C, Green D, et al. Identification of a bilirubin receptor that may mediate a component of cholestatic itch. Elife, 2019,8:e44116

3 Yosipovitch G, Reaney M, Mastey V, et al. Peak Pruritus Numerical Rating Scale: psychometric validation and responder definition for assessing itch in moderate-to-severe atopic dermatitis. Br J Dermatol, 2019,181(4):761-769

4 Liu BW, Li ZX, He ZG, et al. Altered expression of itch-related mediators in the lower cervical spinal cord in mouse models of two types of chronic itch. Int J Mol Med, 2019,44(3):835-846

5 Chen M, Li ZX, Wang Q, et al. Altered Expression of Differential Genes in Thoracic Spinal Cord Involved in Experimental Cholestatic Itch Mouse Model. Curr Med Sci, 2018,38(4):679-683

6 Wang Q, Li ZX, Liu BW, et al. Altered expression of differential gene and lncRNA in the lower thoracic spinal cord on different time courses of experimental obstructive jaundice model accompanied with altered peripheral nociception in rats. Oncotarget, 2017,8(62): 106 098-106 112

7 Liu T, He Z, Tian X, et al. Specific patterns of spinal metabolites underlying alpha-Me-5-HT-evoked pruritus compared with histamine and capsaicin assessed by proton nuclear magnetic resonance spectroscopy. Biochim Biophys Acta, 2017,1863(6):1222-1230

8 Liu BW, Li ZX, He ZG, et al. Altered expression of target genes of spinal cord in different itch models compared with capsaicin assessed by RT-qPCR validation. Oncotarget, 2017,8(43):74 423-74 433

9 Liu T, Li Z, He J, et al. Regional Metabolic Patterns of Abnormal Postoperative Behavioral Performance in Aged Mice Assessed by (1)H-NMR Dynamic Mapping Method. Neurosci Bull, 2020,36(1):25-38

10 Wang J, Zeng HL, Du H, et al. Evaluation of metabolites extraction strategies for identifying different brain regions and their relationship with alcohol preference and gender difference using NMR metabolomics. Talanta, 2018,179(1):369-376

11 Malatji BG, Meyer H, Mason S, et al. A diagnostic biomarker profile for fibromyalgia syndrome based on an NMR metabolomics study of selected patients and 
controls. BMC Neurol, 2017,17(1):88

12 de GraafRA, Chowdhury GM, Behar KL. Quantification of high-resolution (1)H-[(1)(3)C] NMR spectra from rat brain extracts. Anal Chem, 2014,86(10): 5032-5038

13 Guan M, Xie L, Diao C, et al. Systemic perturbations of key metabolites in diabetic rats during the evolution of diabetes studied by urine metabonomics. PLoS One, 2013,8(4):e60409

14 de GraafRA, Chowdhury GM, Behar KL. Quantification of high-resolution (1)H NMR spectra from rat brain extracts. Anal Chem, 2011,83(1):216-224

15 Widerstrom-Noga E, Pattany PM, Cruz-Almeida Y, et al. Metabolite concentrations in the anterior cingulate cortex predict high neuropathic pain impact after spinal cord injury. Pain, 2013,154(2):204-212

16 Sun YG, Zhao ZQ, Meng XL, et al. Cellular basis of itch sensation. Science 2009, 325(5947):1531-1534

17 Sun YG, Chen ZF. A gastrin-releasing peptide receptor mediates the itch sensation in the spinal cord. Nature, 2007,448(7154):700-703

18 Song Y, Pan X, Liu C, et al. Role of nociceptive arcuate nucleus neurons in chloroquine-induced pruritic behaviors in mice. J Huazhong Univ Sci Technolog Med Sci, 2012,32(6):919-922

19 Liu C, Liu TT, He ZG, et al. Inhibition of itch-related responses by selectively ablated serotonergic signals at the rostral ventromedial medulla in mice. Int J Clin Exp Pathol, 2014,7(12):8917-8921

20 He ZG, Liu BW, Li ZX, et al. Altered expression profiling of spinal genes modulated by compound $48 / 80$ in a mouse itch model. J Anesth Perioperat Med, 2017,4(5):220-224

21 Zeng HL, Yu FL, Zhang Z, et al. Quantitative proteomics study of host response to virulent and attenuated pseudorabies virus infection in mouse brain. Biochim Biophys Acta Proteins Proteom, 2018,1866(2):307-315

22 Wang SJ, Lirng JF, Fuh JL, et al. Reduction in hypothalamic ${ }^{1} \mathrm{H}-\mathrm{MRS}$ metabolite ratios in patients with cluster headache. J Neurol Neurosurg Psychiatry, 2006,77(5):622-625

23 Duan B, Cheng L, Ma Q. Spinal Circuits Transmitting Mechanical Pain and Itch. Neurosci Bull, 2018,34(1): 186-193

24 Patti GJ, Yanes O, Shriver LP, et al. Metabolomics implicates altered sphingolipids in chronic pain of neuropathic origin. Nat Chem Biol, 2012,8(3):232-234

25 Johnson CH, Patti GJ, Courade JP, et al. Alterations in Spinal Cord Metabolism during Treatment of Neuropathic Pain. J Neuroimmune Pharmacol, 2015, 10(3):396-401

$26 \mathrm{Ma}$ Q. Itch Modulation by VGLUT2-Dependent Glutamate Release from Somatic Sensory Neurons. In: Carstens E, Akiyama T, editors. Itch: Mechanisms and Treatment. Boca Raton (FL), 2014.

27 Bourane S, Duan B, Koch SC, et al. Gate control of mechanical itch by a subpopulation of spinal cord interneurons. Science, 2015,350(6260):550-554

28 Liu T, Han Q, Chen G, et al. Toll-like receptor 4 contributes to chronic itch, alloknesis, and spinal astrocyte activation in male mice. Pain, 2016,157(4): 806-817

29 Jing PB, Cao DL, Li SS, et al. Chemokine Receptor CXCR3 in the Spinal Cord Contributes to Chronic Itch in Mice. Neurosci Bull, 2018,34(1):54-63

(Received May 26, 2020; accepted June 12, 2020) 